\title{
BIM for Infrastructure: integrating spatial and model data for more efficient contextual planning, design, construction and operation
}

\author{
M. Suchocki \\ Information Systems Panel, Institution of Civil Engineers, UK
}

\begin{abstract}
Global GDP is forecast to double between 2010-2030, with existing civil infrastructure determined unable to handle an anticipated resultant $50 \%$ increase in usage. The global infrastructure investment required across land transport, telecoms, electricity, and water sectors to meet GDP and usage growth is calculated at around $\$ 53$ trillion. This is at a time when the resources required to deliver such investment are similarly coming under pressure.

This quandary of trying to meet increasing infrastructure investment with a shortfall in resource suggests new ways of working needs to be adopted. Emerging Building Information Modelling (BIM) for Infrastructure solutions are extending the industry's capability by focusing on exploiting the value of data rather than the execution of routine activities such as drawing during professionals' daily work. These solutions can provide more efficient infrastructure development, with fewer resources, reduced timescales and improved outcomes.

The paper will illustrate how professionals such as geospatial practitioners, architects, and engineers are beginning to leverage BIM for Infrastructure to collaboratively understand existing urban and natural environments, conceptualise new infrastructure, analyse alternatives, visualise proposals and pass approved options on to full design development. Processes for data continuity through the entire capital project lifecycle into operation will be described and aspects such as interoperability and exploitation of multiple data sources will be covered. Keywords: BIM, infrastructure, mandate, interoperability, geospatial.
\end{abstract}




\section{Introduction}

Infrastructure investment of around $\$ 53$ trillion is forecast over the next 15 years linked to an expected doubling of global GDP [1]. This investment spans across land transport, telecoms, electricity, and water sectors amounting where the existing civil infrastructure is deemed unable to handle an anticipated $50 \%$ increase in usage, let alone a doubling or tripling of passengers and freight during a 20 year period.

The most recent ICE State of the Nation report [2] stated that if the UK is to rise rather than fall in World Economic Forum rankings (currently $28^{\text {th }}$, down from 24th) the approach to delivering and maintaining infrastructure requires attention. The National Infrastructure Plans published in 2010 and 2013 have advanced the way Government and industry engage on infrastructure but gaps remain around ensuring the correct level of investment in maintaining existing assets as well as delivery of new infrastructure. The report also identified that engineering skills are in increasing demand across the UK economy in terms of both the number of engineers needed and the breadth of capabilities.

The Royal Academy of Engineering [3] estimated the cross industry number of UK Science Engineering and Technology (SET) professionals to be $3.6 \mathrm{M}$ with a further 850,000 needed to meet UK demand by 2020 . The resultant need for over 100,000 annual SET graduates is however not served by current UK output of only 90,000 per annum.

Delivering the required infrastructure investment with a constrained resource cannot be achieved without adoption of new working practices and technologies. Construction 2025 [4] delivered a vision of achieving 33\% reduction in costs, 50\% faster delivery, 50\% lower emissions and 50\% improvement in exports to help meet the growing global demand in the construction sector. This was deemed achievable by making improvements in many areas such as the attractiveness of the sector, BIM adoption, embracing sustainability and clear leadership.

BIM is clearly a key enabler of change and can provide a high proportion of the improvements if correctly adopted. However within the infrastructure sector, BIM remains something of an enigma with poor understanding of the technology as well as the processes to be used in its application.

\section{What is BIM for Infrastructure?}

The ICE Information Systems panel [5] and BIM Action Group view BIM as the purposeful management of information through the whole life cycle of an infrastructure asset. It necessitated adopting a managed approach to collecting and using information across the life-cycle of a built asset, using technology throughout for the preparation of graphical models and associated information.

The 'managed approach' is perhaps the most important concept to understand as it introduces the greatest process change into design, construction and operation of a built asset. Simply using new '3D' technology in design does not provide BIM, it is instead principally down to controlling who prepares information, how it is prepared, what gets prepared and at what phase; that is what provides the 
foundation for BIM. Moreover, it is only by changing the process of designing, constructing and operating assets that significant benefits can be achieved, otherwise only incremental improvements can be delivered as the majority of work gets delivered using traditional approaches with no opportunity for improvement; you can't keep doing the same thing and still expect a different result.

\subsection{How should BIM be applied to projects?}

There are clearly multiple options available to a design and construction team when selecting the BIM technologies for an infrastructure project. Adopting good practices coupled with industry standards is a logical recommendation and may be a requirement when working for certain public or private clients.

For example the UK Government BIM initiative has sought to introduce BIM in order to add value to centrally funded public sector investments. The initiative has seen all parts of the industry engaged and led to a significant amount of supporting material being developed. For projects the most important guide is PAS 1192-2:2013 [6], which details how BIM can be applied to a capital investment irrespective if it is a building or infrastructure scheme.

Determining what information is required by phase is the starting place and the PAS 1192:2 fundamental principles of Level 2 information modelling include:

a) originators produce definition information in models which they control, sourcing information from other models where required by way of reference, federation or direct information exchange;

b) provision of a clear definition of the employer's information requirements (EIR) and key decision points (as shown in Figure 1);

c) evaluation of the proposed approach, capability and capacity of each supplier, and their supply chain, to deliver the required information;

d) a BIM execution plan (BEP) that includes a master information delivery plan (MIDP);

e) provision of a common data environment $(\mathrm{CDE})$ to store shared asset data and information;

f) application of the 'Level 2' processes and procedures;

g) information models developed using a mixed set of enabling tools.

This is all applied against a structured information delivery lifecycle that seeks to have a body of information grow over time, rather than a typical saw-tooth of information creation and recreation caused through poor collaboration between participants and across phases of work.

\section{An approach for infrastructure}

Applying the PAS 1192:2 principles to a typical infrastructure project requires confirmation of the type of information that needs to be created by phase and by participant. This should be coupled with understanding the art of the possible and potentially introducing radical change to the design and construction process to leverage opportunity from new technologies and ways of working. 


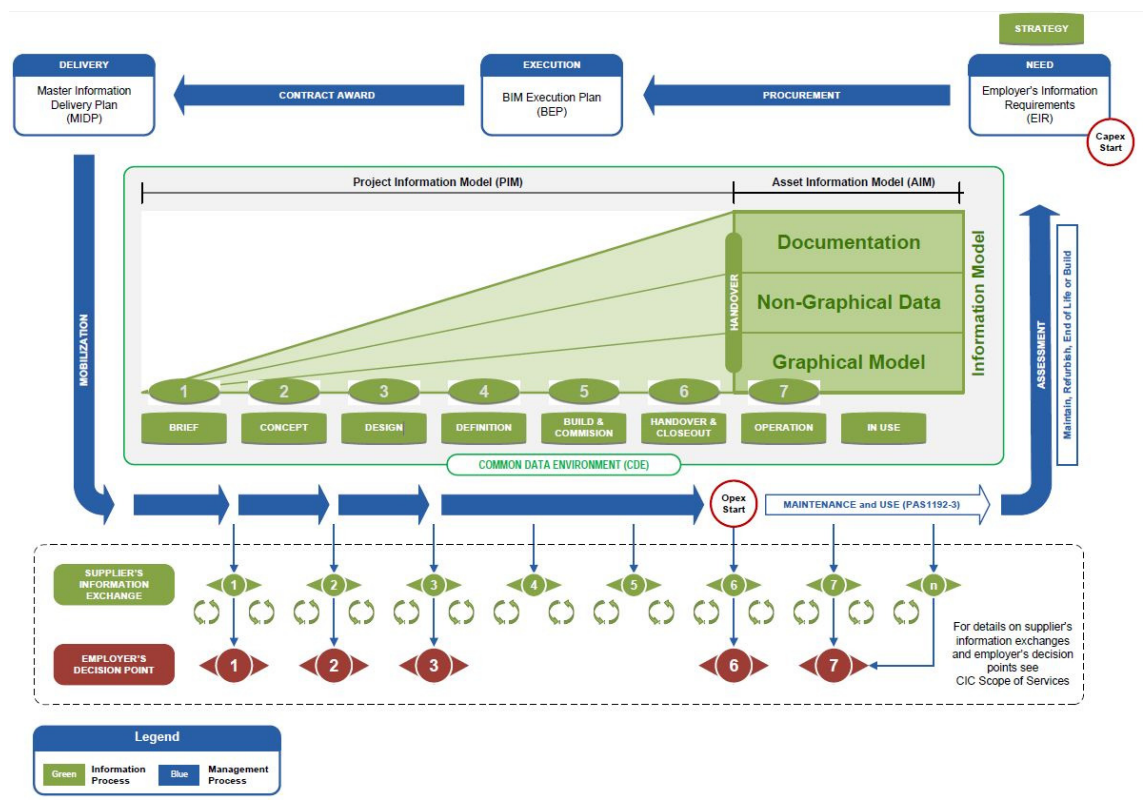

Figure 1: The PAS 1192:2 information delivery lifecycle.

Contractual and commercial implications may play a part where specifically defined obligations for roles under say the NEC3 [8] family of contracts or from the Construction, Design and Management (CDM) Regulations [9] need to be performed; the CIC BIM Protocol guidance [10] should be applied to identify what if any modifications are required to contracts or role profiles when adopting BIM.

Collaboration between disciplines has historically suffered from poor ability to share data from one application to the next. This results either in incomplete or incorrect data sharing or a need to fully remodel a design. It is therefore important that a project team use the PAS 1192:2 principles and/or apply the CPIx Protocol [11] to identify the technologies, data formats and workflows across disciplines and phases to mitigate data exchange issues from the outset.

\subsection{Integrating BIM and GIS during early project development}

Infrastructure projects uniquely can start by leveraging available data for an area under inspection that is either owned by the client, in the public domain or can be acquired from a data provider. For example Ordnance Survey in the UK offers data sets that include:

- $\quad$ raster data at a range of scales

- terrain data at a range of scales

- topographic data that now includes building heights

- $\quad$ postcode boundary coverage data

- vector data of urban and rural environments 
Such data can be immediately used, searched, filtered and displayed within GIS and engineering software to gain an understanding of a region. This data can be supplemented with further coverage data such as land ownership, sites of special scientific interest, pre-determined corridors, flood extents, and aerial photography.

Local surveys frequently need to be undertaken in the region of preferred options to supplement the geospatial data sets. The data can be acquired using traditional techniques such as field surveying with total stations to accurately record positions of specific structures, edges of highways, manhole locations or natural items such as extent of watercourses, trees or hedgerows. Increasingly point cloud data acquisition is being used from laser scanners or through photogrammetric image transformation taken either at ground level or with aerial views from UAVs (unmanned aerial vehicles) or aircraft. This point cloud data can be readily used within many software platforms to provide a highly accurate real world context to supplement transformed geospatial data sources and early conceptual design proposals.

A number of technologies can now transform this comprehensive mix of base data to create three-dimensional representations such as in Figure 2, of a region applying realistic styles to buildings or converting single vectors into realistic roads, rail lines or utility pipe networks. Designers and engineers can then plan new infrastructure or modifications to existing against a full context without necessitating local detailed surveys until early options have been confirmed or eliminated against known constraints.

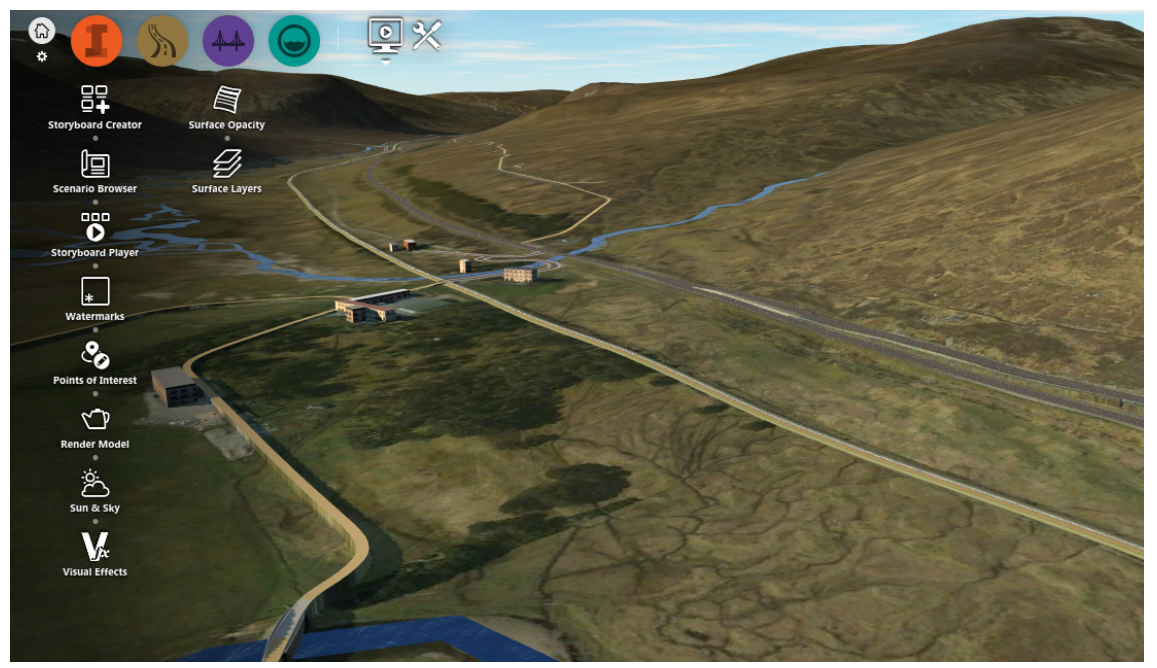

Figure 2: Autodesk InfraWorks ${ }^{\circledR}$ existing condition model prepared using geospatial source data prior to conceptualising new road options.

The latest technologies also allow for concept design options to be analysed and infrastructure proposals to have immediate engineering rules applied constraining proposals to those that are technically viable. For example such early 
optimisation for highway vertical alignments, junctions, bridges, surface water drainage and culverts can be carried out within Autodesk's InfraWorks ${ }^{\circledR}$ technology. This approach immediately reduces downstream effort by focusing on appropriate design options from early phases as well as carrying out preliminary analysis.

Preferred option concept models can be used within design review meetings, planning consultation activities, shared with stakeholders for viewing on the Internet or on mobile devices. This can confirm the appropriateness of proposals and significantly improve potential for planning approval.

\subsection{Model data during detailed design}

Once preferred options have been approved, projects will move into one or more design phases. For infrastructure, design has historically been dominated by two dimensional views in plan, elevation of cross-section of a scheme. A threedimensional model of a proposal would typically have been developed for a highway or rail alignment, however this would not have been directly linked to the cross-sections that would have been developed using calculation at specified intervals and requiring complete rework should the profile change.

Civil BIM solutions now provide the ability to create rail profiles, engineering structures, highway alignments and go beyond simple 3D geometry to integrate to other required model views as well as holding or calculating further attributes for sections or point locations. Such models can be created from simple alignments, such as the options developed during conceptual designs, and enhanced to apply all engineering constraints needed for a viable scheme including; horizontal profile, vertical profile, junctions, super-elevation, cross-section changes, drainage and structures. These fully developed designs can then be exported for re-integration into a fully aggregated view with the geospatial and local survey data used during initial option selection.

Where structures and buildings are defined within an infrastructure scheme, the same mix of technologies used within vertical building projects can be applied. These BIM technologies are generally more mature with a number of vendor solutions available for architectural modelling, structural design and analysis, building services design and analysis, data aggregation and visualisation.

\subsection{Construction and operation}

BIM technology is fully applicable within the construction and operational phases of built assets. The previously described design and geospatial technologies as well as prepared data need to be utilised but also supplemented with solutions for asset data capture and management. Increasingly mobile field based solutions are being leveraged to ensure the data is available or can be directly updated at the work site.

Linkage of model data developed during design into construction execution permits significant improvements in productivity and accuracy include setting-out positioning based on model coordinates and plant automation typically using GPS rather than ground based positioning. This eliminates a significant amount of 
setting-out effort such as placing markers, profile boards or road-pins (now forbidden for use on Highways England schemes) and the associated risk of errors. Following construction, verification of as-built vs. designed can also be executed using capture of specific points or using laser scans.

Progress reporting using mobile field based reporting, laser scanning or photo/video captured by UAVs is increasingly being adopted, particularly for large projects such as infrastructure schemes. Actual project progress can also be compared against four-dimensional sequence simulations prepared from models linked to project plans.

Field based data capture is significantly accelerating the time for snagging and commissioning activities. Use of flexible forms allows any required information to be checked or recorded on mobile devices, supplemented with photographs and linked to specific locations or objects within a model.

This same approach is being adopted by facilities and asset managers to take data into the field when carrying out inspections as well as planned or reactive maintenance. Asset managers are also able to identify data omissions or errors that need correcting to have a complete overview of managed assets. This working approach is no longer limited to models associated to single large assets e.g. buildings or structures, but can now work across long infrastructures enabling civil owner operators to leverage the same benefits.

\subsection{Data management}

The common data environment requirement within PAS 1192:2 is crucial to correctly managing information through both the capital and operational phases of infrastructure assets. This is not necessarily a single technology, however clarity over what constitutes the primary source of truth needs to be determined by the participants in the development or operation of assets.

An infrastructure project may have data stored in one or more geospatial systems, on network drives, document management systems and in internet based collaborative working platforms. Data from a mix of these sources will be leveraged during design development and the users need to be confident of the origin of the data, the version and its appropriate usage.

The transition of data from work in progress, to shared and, once deemed appropriate for a given use, to published is a principle that all project participants must understand. Where data is stored and in what formats should also be clarified to avoid risks such as data duplication, misuse, or lack of use.

\subsection{File formats and interoperability in relation to BIM for Infrastructure}

\subsubsection{Infrastructure design}

Typical civil design 3D data formats include DWG, DGN, GENIO string files and LandXML. These are not specifically BIM formats but do permit geometry to be transferred between different vendor applications to recreate designed alignments and carry out subsequent design procedures. Some formats can also include additional attributes linked to specific discrete objects or object families. 
The use of open standards is desired by many clients and project teams to avoid prescribing proprietary formats and increasing choice of technology solutions. Within the infrastructure sector it is unlikely that any single format can be adopted due to the large variety of disciplines that contribute to scheme development.

The E57 file format is supported by many software platforms as it provides a compact, vendor-neutral form for storing point clouds, images, and metadata produced by $3 \mathrm{D}$ imaging systems, such as laser scanners or from photogrammetric processing of image data captured by ground based digital cameras or UAVs.

ISO15926 is an international set of standards for the sharing, exchange, and hand over of information associated with the engineering, construction, and operation of industrial assets typically process or power plants. This has been applied effectively for the preparation of standard catalogues that allows all manufacturers to provide their information in an open common format that can be exchanged into proprietary formats of existing 3D systems.

Industry Foundation Classes (IFC) from buildingSMART where version IFC4 has been registered as ISO 16739:2013 [12] can allow geometric and attribute data to be transferred between different technologies consistently. The IFC schema is mature within building design however the support for infrastructure is currently limited to solid model or surface geometry exchange; this will improve in coming years as there is a joint buildingSMART and Open Geospatial Consortium (OGC) initiative underway to develop IFC alignment (version 1.0 was released in March 2015), IFC bridge and IFC road definitions.

LandXML is an XML data file format containing civil engineering and survey measurement data commonly used in the Land Development and Transportation Industries. It was developed over a decade by a community of volunteer organisations and individuals with schema version v1.2 published in 2008. The LandXML.org organisation is currently working on LandXML v2.0 to incorporate $3 \mathrm{D}$ road model improvements, enhance 3D/volumetric parcel support, improve intelligent construction data flow and add flexible road template definitions.

The Finnish transport authority in 2014 specified that data submissions on projects would use the LandXML v1.2 Inframodel 3 (IM3) extension that provides additional aspects such as missing descriptive information, source data attributes, design parameters, cross section properties, landscape features, pipe networks and street furniture. IM3 is also the baseline for buildingSMART's Model View Definition for LandXML.

COBie as described within BS1192:4 [13] is an open schema that can be used to transfer data from a design for validation processes and additionally population of asset management systems. COBie can be mapped to infrastructure but there are currently no live examples where this format has been used to exchange data during a major infrastructure project or consistently into operational activities for an asset owner. There are however efforts underway, principally led by the UK Environment Agency, to determine infrastructure client data requirements during a project lifecycle and into operation, which are then mapped into a COBie 'template' that the supply chain must seek to populate either automatically from BIM technologies or with manual data entry. 
Interim Advice Note 184/14 [14] from Highways England published in 2014 is a draft standard for BIM and CAD data use. It acknowledges that challenges exist for the supply chain in the adoption of BIM and that HA will need to adopt a pragmatic but progressive approach to BIM. However CAD and model formats previously referred are stated as appropriate with little concern over data interoperability where the process has been clearly defined by a project team.

\subsubsection{Geospatial formats}

Geospatial applications use and generate a range of raster and vector data. This data can be provided as files or increasingly served from databases where information is only provided for a specified area under inspection or query.

Formats developed by the OGC are very widely adopted within the geospatial sector and include the Geography Markup Language Encoding Standard (GML), Web Map Service (WMS), Web Feature Service (WFS) and CityGML. OGC data formats are the basis for Ordnance Survey Mastermap as well as other commonly available datasets.

Interoperability challenges from the use of geospatial formats are relatively rare, with the majority of applications able to read and create both proprietary and open data formats.

\section{Resource challenges}

As identified in the RAE Jobs and Growth report [3] there is an anticipated resource shortage across Science, Engineering and Technology sectors that will benefit from but also negatively impact BIM adoption. BIM is however proving an attractive capability for existing and new entrants into the construction sector. Application of innovative technology, structured data usage and efficient processes permits the construction industry to position itself as a sector that can retain employees and encourage others to join.

It is particularly important to attract young people into the industry at school and undergraduate level. There are efforts in progress by professional bodies such as the Institution of Civil Engineers [15], individual companies and technology vendors [16] to raise the profile of the sector using BIM as a key factor to differentiate how the industry operates today.

There remain challenges with BIM education as professional accreditation has yet to embrace BIM as a required capability. As a result University undergraduate tuition of BIM is inconsistent. The BIM Academic Forum (BAF), a sub-group of the UK BIM Task Group, has undertaken an exercise to understand how BIM is currently taught in courses [17] revealing a range from well-developed course structures linked with industry to no awareness of the topic by some.

The BAF has also worked to develop a framework for required outcomes for any organisation delivering BIM tuition in association with the BIM Task Group [18]. As these recommendations get adopted there is likelihood that, coupled with the forecast high investment in infrastructure schemes, the construction industry will develop into a front running sector in a new digital economy encouraging and attracting young people to join. 


\section{Summary}

BIM for Infrastructure is not an aspiration; it is a managed process for leveraging available technology to understand the context of a project, explore and assess options, fully design preferred proposals and efficiently execute the construction, providing a comprehensive accurate set of data to the owner and operator of the delivered assets. It increases certainty, better meets client expectations, allows for more rapid execution and, because of process benefits such as elimination of errors, proves cost effective.

The temptation to immediately start engineering solutions in familiar technologies should be avoided; project teams should instead apply best practices such as the guidance prepared for the UK Government BIM initiative to firstly plan a project by identifying what information is needed, when and by which party. The clarified requirement will then help the selection of the specific technologies to be adopted.

The rapid innovation occurring in the technologies for construction should be considered as an enabler and driver of change. Using traditional tools and processes eliminates the opportunity for any improvement because only known workflows and outcomes can be achieved. Although adopting new ways of working does introduce risk due to lack of familiarity and confidence in the approach, provided the process of adoption is managed and monitored, these risks can be controlled.

Determining the data formats frequently causes project teams' confusion and concern. During the execution of projects it is entirely reasonable to adopt proprietary formats determined by the identified data requirements and chosen mix of technologies during the initiation phase of a project. Where there are concerns with specifying proprietary formats e.g. data submission requirements by a client or identified issues with exchanging data between systems, open formats should be used wherever they are appropriate. The specification of data and file formats, both open and proprietary, does require careful consideration to avoid introducing additional complexity, effort and risk of error.

\section{References}

[1] Strategic Transport Infrastructure Needs to 2030, OECD, March 2012.

[2] State of the Nation, Infrastructure 2014, Institution of Civil Engineers, London, June 2014.

[3] Jobs and growth, Royal Academy of Engineering, London, September 2012.

[4] Industrial Strategy: government and industry in partnership, HM Government, July 2013.

[5] ICE, https://www.ice.org.uk/about-us/what-we-do/information-systemspanel

[6] PAS 1192:2 Specification for information management for the capital/delivery phase of assets using building information modelling, the British Standards Institution, February 2013. 
[7] Government Soft Landings, http://www.bimtaskgroup.org/gsl/

[8] New Engineering Contract, https://www.neccontract.com/

[9] The Construction (Design and Management) Regulations 2015, Crown copyright 2015.

[10] Outline Scope of Services for the role of Information Management (the BIM Protocol), Construction Industry Council, London, February 2013.

[11] CPIx Protocol, http://www.cpic.org.uk/cpix

[12] ISO 16739:2013, Industry Foundation Classes (IFC) for data sharing in the construction and facility management industries, ISO 2013.

[13] BS1192:4, Collaborative production of information; Part 4: Fulfilling employer's information exchange requirements using COBie - Code of practice, The British Standards Institution, September 2014.

[14] Interim Advice Note 184/14, Highways Agency Data \& CAD Standard, Highways Agency, August 2014.

[15] ICE's Manifesto for Infrastructure 2015, Institution of Civil Engineers, London, 2015.

[16] Autodesk Education Community, http://www.autodesk.com/students

[17] Current Position and Associated Challenges of BIM Education in UK Higher Education, BIM Academic Forum, March 2015.

[18] Learning Outcomes Framework v1.0, http://www.bimtaskgroup.org/ education-and-training/ 